\title{
Medicalización y disciplinamiento. La construcción higienista del espacio femenino, 1850-1920
}

Manuel Durán Sandoval ${ }^{1}$

En el presente trabajo intentaremos establecer algunos lineamientos respecto al discurso médico higienista y los imaginarios corporales/espaciales impuestos a las mujeres según el ideario político liberal del siglo XIX. En este sentido, las mujeres debían ser activas en la construcción de la "República" y contribuir a la educación de los ciudadanos, pero en este vértice ellas mismas quedaron excluidas de la ciudadanía, siendo recluidas en los gineceos como devotas madres y amantes esposas. La ciencia y la ideología se asociaron justificando natural e ideológicamente la "dominación masculina."

El discurso médico-higienista estableció hacia mediados del siglo XIX en Chile un corpus normativo sobre el cuerpo de hombres, mujeres y niños, estableciendo dinámicas de exclusión y ritualidad. En el centro del debate se situó el cuerpo de las mujeres como geografía de signación. Este proceso abarcó todos los ámbitos de desarrollo nacional como la medicina, la educación y el desarrollo urbano. En el presente ensayo se abordarán algunos de estos ámbitos con el fin de determinar los mecanismos coercitivos utilizados por el discurso liberal y científico en su proceso de normalización del cuerpo y los espacios femeninos.

\section{La constitución del movimiento médico higienista y la regulación del espacio}

Entendemos por "Higienismo" una corriente de pensamiento abocada al estudio de problemáticas demográficas y poblacionales y con especial atención a grupos de presión social como mendigos, prostitutas y alcohólicos. Este pensamiento se estableció hacia me- 
diados del siglo XIX siendo uno de sus precursores el argentino Guillermo Rawson, quien inauguró en 1873 la cátedra de higiene en la facultad de medicina de la Universidad de Buenos Aires. En 1881 publicó un estudio sobre higienismo titulado "Observaciones sobre Higiene Internacional" contribuyendo a la difusión de las ideas higienistas en toda Latinoamérica. En Chile estas teorías fueron difundidas por el pensador y científico prusiano Rodolfo Amando Philippi quien publicó en 1866 su obra "Elementos de Historia Natural." ${ }^{2}$ Pensadores liberales como Philippi y Barros Arana impulsaron el estudio de las ciencias, siendo éstas incluidas en la Ley Orgánica de Instrucción Secundaria y Superior de 1879, la cual garantizaba la autonomía de los catedráticos y profesores en materia científica. La institución del discurso científico como "episteme" constituyó, prontamente, una casta médico-higienista preocupada de las problemáticas sociales. En la Revista Médica se define a la 'hijene' como una de las más importantes aplicaciones del médico en su rol de 'terapeuta': "La hijene no es otra cosa, que una de las mas hermosas aplicaciones del arte médico..." 3

El período coincide con la formación de una elite médico profesional que tiene su mayor representación en la Escuela de Medicina de la Universidad de Chile, la Sociedad Médica de Santiago y la Revista Médica de Santiago ${ }^{4}$, definida esta última como el órgano difusor de la clase médica: "Ese periódico procurará la difusión de la ciencia, (...) la generalización de la nociones mas indispensables de higiene pública, i privada; él hará conocer el estado sanitario del país, i se aplicará a dilucidar las cuestiones mas importantes de salubridad pública. ${ }^{\prime 5}$

Muchos de los miembros de la comunidad médico-científica accedieron a cargos políticos como es el caso del médico Guillermo C. Blest, quien se desempeñó como diputado y senador entre 1831 a 1876. Destacada fue, también, la participación del médico José Joaquín Aguirre Campos, Decano de la Facultad de Medicina de la Universidad de Chile, gobernador y diputado por Los Andes y Santiago. ${ }^{6}$

De esta forma el discurso higienista se integró, prontamente, a las políticas institucionales adquiriendo ribetes valóricos que lo situaban como agente normalizador; así se señala en un artículo titulado "El Rol del Médico": "La conservación de la salud, su restablecimiento, ¿son los únicos objetos que el verdadero médico 
debe tener en vista? (...) ¿no tiene también, como el sacerdote que conduce a los mortales por el camino de la moral...?"7

Términos como disciplina y saneamiento eran la propuesta ante lo que los médicos denominaban una corrupción del cuerpo y del alma. El higienismo, por tanto, no velaba únicamente por el bienestar individual, sino por el desarrollo y la estabilidad de la nación entera cuya base se sostenía en el cuerpo y los hábitos femeninos. Pero esta relevancia ocultaba un doble discurso, la mujer a quien se le concedía un rol importante en la construcción del sistema ideológico como madre, no tuvo mayor injerencia en la formulación de los discursos científicos y políticos, siendo excluida de ejercicio de las ciencias y el conocimiento institucional; así lo constata Eloísa Díaz en sus memorias para optar al grado de Licenciada en la Escuela de Medicina de la Universidad de Chile: "Vedado estaba a la mujer chilena franquear el umbral sagrado del augusto templo de las ciencias." ${ }^{8}$ La mujer se encontraba excluida del ejercicio científico y médico por considerase contrario a su naturaleza, en el texto anteriormente citado se señala: "Hai reacios que piensan que la mujer, haciéndose médico, pierde los rasgos de su carácter, para varonilizarse i abdicar así de las prerrogativas de que goza en la sociabilidad."

\section{Disciplinamiento del cuerpo y maternidad}

El proyecto ilustrado pretendía sumergir a la sociedad en campos de normalización, estableciendo a la economía como "sintagma de lo biopolítico" con el fin de maximizar la productividad de los cuerpos ${ }^{10}$. En este contexto el cuerpo femenino se constituyó en un espacio en disputa entre las agencias reguladoras de la vida y los celadores del alma. Las agencias de normalización establecieron un programa correccional del cuerpo y el espacio, ${ }^{11}$ estableciendo en 1824 la Casa de Corrección para mujeres. ${ }^{12}$ Dicho establecimiento fue restablecido a petición del pensador Manuel de Salas quien obtuvo del ministro del Interior, Francisco Antonio Pinto, una subvención derivada de las multas en la Corte de Apelaciones, para la disposición de una casa correccional femenina. En la Casa se estableció un régimen de trabajo conventual, incitando a las internas a la rehabilitación de delitos tan variados 
como la mendicidad, la prostitución y el amancebamiento. En un documento de la Gaceta de los Tribunales fechado el 9 de marzo de 1844 se consigna que Ignacia Flores es condenada a "vergüenza pública" y a diez años de trabajos forzados y reclusión por el delito de "alcahueta de su hija." ${ }^{13} \mathrm{El}$ recinto contaba con un dormitorio donde se alojaban 44 mujeres y 12 niños y al igual que en la mayoría de las Casas de Internamiento las jornadas de trabajo iban acompañadas por sesiones de instrucción cristiana. Las Casas de Maternidad y Hospicios se instituyeron en casas de rehabilitación, donde se reeducaban a los sujetos con trabajo y mortificación. Así lo señala el Médico Ramón Allende en su discurso en la Escuela de Ciencias Médicas de la Universidad de Chile: "En esas casa de maternidad (...) bajo el mas inviolable sigilo i sin temor de ninguna especie, podría la desgraciada joven pagar su tributo al dolor pero evitar la deshonra que la sobrevendría." 14

Gran parte de estos establecimientos eran solventados gracias a la caridad de particulares y la administración de instituciones religiosas, así lo fueron el Asilo del Salvador fundado el 18 de octubre de 1844 e ideado para refugio de mendigos y menesterosos y el Hospital San Juan de Dios heredero del Hospital de Nuestra Señora del Socorro creado en $1556 .{ }^{15}$

Era imprescindible que el Estado se hiciera cargo de las problemáticas de asistencia. En 1842 el diputado Pedro Palazuelos planteaban ante la sesión de los Cuerpos Legislativos del Congreso la urgente necesidad de que fuera el Estado y no la buena voluntad de vecinos ilustres o instituciones religiosas los que se hicieran cargo de los sectores marginados de la sociedad: "Si el Estado no se encarga de ellos (...) temo que la clase indigente se levante contra la sociedad y asalte para subsistir."16 Con este objetivo el Gobierno Nacional creó una comisión de seis señores ilustres para administrar al hospital San Juan de Dios, dicha política de asistencia quedó en manos de una Junta Central de Beneficencia y Salud Pública. Finalmente se dictó en 1886 el reglamento general para las juntas de beneficencia, regidas por un sistema uniforme con semiautonomía, aunque dependiendo del Ministerio del Interior. Todo esto contribuyó a consolidar la tendencia higienista entre los círculos aristocráticos del país, fortaleciendo el sistema de asistencia destinado a combatir la pobreza y la mendicidad. Con este objetivo se fundaron la Casa de Locos 
(1856), Casa de Orates (1883), Casa de Expósitos (1856) y la Casa de Maternidad (1870). Ejemplo de este proceso de asitencialismo lo encontramos en el discurso proferido por el médico Ramón Allende en la Facultad de Medicina de la Universidad de Chile en 1865, en el cual enfatizaba la necesidad de establecer casas de maternidad destinadas al auxilio de quienes careciendo de medios de asistencia o, simplemente, por "accidentes especiales" hayan sido abandonadas: "Las casas de maternidad son establecimientos públicos destinados a recibir toda parturienta que, por su falta de recursos o accidentes especiales, no pudiera asistirse en su hogar. Basta esto para que ya comprendamos su objeto i aplicaciones."17

La Casa de Maternidad no sólo debía acoger, sino también prevenir el delito: "I no es esto solo; hai algo mas grande todavía y mas trascendental, ese establecimiento, esa casa de maternidad, cuya existencia es una necesidad imperiosa, seria una barrera contra el crimen; la mujer teniendo ese amparo esperaria sin terror la hora de su alumbramiento y jamas atentaria contra su hijo..."18

Otro de los objetivos que se perseguía con el establecimiento de este tipo de instituciones era implementar una "política de la maternidad" reduciendo los índices de orfandad por abandono: "Con ese establecimiento se evitaría otro crimen no menos atroz, la esposicion; la esposicion, comprendeis, señores, lo que es un crimen horrible resultado tan solo de la miseria y desesperacion de una pobre madre." ${ }^{\prime 19}$

La mujer, bajo este criterio, se encuentra en una situación de riesgo social, así lo señala Allende en el documento anteriormente citado: "Vereis con cuanta facilidad puede romperse la armonía, que constituye la razón, en un cerebro poco educado, i afectado violentamente por mil sufrimientos, vereis como os explicáis una locura momentanea que arrastra a la mujer, a la madre a destruir su propia concepción, su propio fruto y mancharse con la infamia." 20

Dado su intelecto débil y ánimo voluble la mujer se encuentra expuesta a los desórdenes y la locura, es deber del Estado, en consecuencia, en su calidad patriarcal fiscalizar a todos aquellos sujetos de la feminidad.

Otro de los males a combatir fue el alcoholismo y la prostitución. La primera ordenanza del 17 de mayo 1892 regulaba la Ley 
de Patentes y Expendio de Alcohol, estos negocios debían cerrarse a medianoche en las ciudades y al atardecer en el campo. En 1902 el Estado aprobó la ley 1515 que regulaba los horarios de las licorerías. La lucha contra el alcoholismo y la prostitución era considerada una cruzada moral. Así lo constata un artículo publicado en el boletín "El Despertar de los trabajadores" el domingo 24 de abril de 1921: “...los culpables de esta vergüenza nacional, repetimos, es la torpe sociedad en que vivimos, la sanguijuela capitalista que se alimenta y vive experimentando hasta la última gota de sudor de nuestras mujeres, sin importarles nada del decaimiento de la raza y el mal nombre que por esta causa hemos conquistado en los diferentes pueblos del mundo."21

Tanto el alcoholismo como la prostitución eran percibidos como agentes degenerativos de la raza y la nación, en otro artículo del 25 de junio de 1921 titulado "Las Orgías del Alcalde (...)," la degeneración moral involucraba a la política, el alcoholismo y la prostitución: "las puertas de las cantinas y de los burdeles los están recibiendo diariamente, todos los dueños de prostíbulos, para granjearse la benevolencia de los alcaldes, los invitan hoy en uno, mañana en otro, haciendo banquetes rodeado todas las rameras se convierten en libidinosas orgías, mientras el champagne corre a torrentes todo en aras de la inmunidad..."22

La prostitución fue percibida como una "virulencia" que aquejaba a las mujeres abandonadas o poco instruidas; rescatarlas era un deber nacional: "debemos llegar hasta aquellos antros del vicio llamados prostíbulos, donde han caído nuestras hermanas envueltas en las redes de la miseria, viciada por la maldad y el egoísmo. Debemos llevar hasta ellas nuestra palabra de amor, tendiéndole nuestras manos y arrancarlas del vicio... debemos ser sus guías, estrecharlas en nuestro corazones olvidando su pasado porque ellas no han sido culpables, han sido víctimas..."23

El médico de la Universidad de Chile Ramón Allende calificaba a la prostitución como un mal derivado del abandono de las mujeres a sus propias pasiones, atentando con ello contra su esencia maternal: "Frecuentemente, por desgracia, hai mujeres que se encuentran en una situación fuertemente excepcional, resultado de su vida libre y abandonada (...) de una conducta libertina y perdida, arrastrando su vida en la crápula mas inmunda i en medio de la orjía; prostituida, en fin a perdido su corazon, sus instintos maternales..."25 
Según estos conceptos la mujer corrompida y abandonada niega a su cuerpo el estado natural de sus instintos como madre, era necesario pues, educarlas para el noble fin de la maternidad. El médico Luis Calvo Mackenna, director de las Gotas de Leche del Patronato de la Infancia señalaba en un instructivo sobre maternidad: "Las madres ignoran la manera de criar y cuidar a sus hijitos, porque generalmente nadie que tenga la preparación necesaria, se ha preocupado o a tenido ocasión de darles los indispensables consejos que esas madres siempre deben conocer." ${ }^{25}$

La mortalidad infantil se constituyó como la mayor preocupación del movimiento higienista, así lo atestigua el médico Victor Körner en el primer Congreso nacional de Protección de la Infancia: "La mortalidad infantil es un hecho que ha principiado a preocupar seriamente la opinión pública en nuestro país (...) en nuestros hombres dirigentes y en nuestro cuerpo médico. ${ }^{126} \mathrm{La}$ causa principal de este mal fue atribuido a la negligencia e ignorancia de las madres: "Cuando se estudian las causa susceptibles de hacer variar las causas de la mortalidad infantil de un país o de una ciudad, se apercibe que, mucho más que ninguna epidemia la ignorancia de las madres y la ausencia de vigilancia de los recién nacidos son los factores que contribuyen a mantener una mortalidad elevada." 27

En este contexto era indispensable generar los mecanismos necesarios para asegurar la protección y control de las nuevas generaciones. A este respecto Vicuña Mackenna señala: "Es la IGNORANCIA, esa nodriza maldita que amamanta todavía los pueblos del Nuevo Mundo, el origen único tal vez de este desorden radical, que consume nuestras sociedades, encendiéndose en su seno una guerra interminable, de las malas pasiones contra la virtud, de la holgazanería contra el trabajo, de la ignorancia, en fin, ciega, implacable y feroz, contra todo lo que tiene por base la civilización y la religión misma." ${ }^{28}$

\section{Higienismo y educación femenina}

El discurso higienista señalaba como foco de virulencia la falta de instrucción femenina, Luis Amunátegui, connotado personero de la educación chilena, considera la educación femenina como un sostén del alfabetismo nacional: "Si en Chile se hubiera hecho a favor de las mujeres respecto a instrucción tanto como se 
ha trabajado en provecho de los hombres, todos los niños sabrían leer, escribir y contar, porque las mujeres son las maestras natas del hogar doméstico." 29

En este mismo lineamiento Eloísa Díaz sostiene: “...reconociendo a la mujer un derecho que naturalmente posee: instruirse para instruir a sus hijos." ${ }^{\prime 30}$

La reforma educacional se situó como prioritaria dentro de los objetivos del movimiento higienista, así se señala en el Informe elaborado por el doctor Murillo a petición del Ministerio de Instrucción Pública: "El deseo de contribuir, en cuanto me fuera permitido, a una reforma o una modificacion que urjentemente reclamaba nuestro plan de estudios i nuestro sistema actual para prevenir los frecuentes i desgraciados males que palpamos consecuencia de la viciosa direccion que se ha dado hasta ahora a la enseñanza." ${ }^{31}$

Durante las primeras décadas del siglo XIX el Estado no se preocupó particularmente de la enseñanza quedando ésta en manos de órdenes religiosas. Los primeros centros educacionales femeninos estuvieron bajo la dirección de la Congregación de la Providencia, invitadas a nuestro país por el gobierno de Manuel Montt y, aunque, la Constitución de 1833 estipulaba un plan general de educación este no se cumplió hasta 1842 con la fundación de la Universidad de Chile, ésta se convertiría en la Superintendencia de Educación, un año más tarde se emitió un decreto por el cual se regularizaba la educación secundaria en nuestro país, la que tiene su eco definitivo en la Ley de Enseñanza Secundaria de $1879 .{ }^{32}$

La segunda mitad del siglo XIX los liceos femeninos fiscales hicieron su aparición tímidamente adoptando el plan de estudios elaborado por Juana Gremler en 1893, en dicho plan de estudios las estudiantes debían ser preparadas para desempeñar su rol como esposas y madres. De esta forma se incentivaban los trabajos domésticos denominado en el "Reglamento para los Liceos de Niñas" de 1900 como Economía Doméstica e Higiene del Hogar. En tanto la educación masculina giraba principalmente en torno a los ramos matemáticos y un ramo de higienismo similar al impartido en los liceos de niñas pero adscrito, en este caso, al ámbito científico de la malla curricular llamado Higiene e Historia Natural. El plan de estudio para las niñas se abocaba a su preparación como madres y no para el desarrollo intelectual, 
así lo manifiesta Eloísa Díaz: "Sensible como mujer por estructura, tímida por consecuencia de su sensibilidad especial, acató ella inconsciente la prohibición injusta que se le imponía i temió traspasar la línea que se le señalara como límite a su actividad social i al desarrollo de su intelijencia." 33

Muchos de los ramos denominado de "adorno" como el canto, música, pintura y dibujo, eran considerados accesorios indispensables en el desempeño de las mujeres en el matrimonio; así lo enfatiza el "Prospecto del Liceo de Niñas No 2 de Santiago: “...se cultiva el sentimiento estético por medio de la enseñanza del canto, del dibujo i de la pintura; se prepara a las alumnas para las necesidades de la vida diaria por las clases de labores de mano, i se despierta de este modo el gusto por ocuparse de estas artes, que a la vez que constituyen un precioso adorno, son un benéfico contrapeso contra los peligros de una vida ociosa, i finalmente se atiende en especial al desarrollo físico por medio de la jimnástica." 34

Claramente la educación femenina se encontraba abocada más al gobierno doméstico que al público. Juana Gremler protestaba contra la exagerada importancia dada a los ramos de adorno que entorpecían la formación del carácter: "Resulta que los ramos técnicos i de adorno tienen en el nuevo plan una importancia desmedida para un Liceo de Niñas, que sólo tiene 8 años de estudio i debe perseguir otros fines que el de dar a las alumnas cierta habilidad manual. Lejos estoi de negar la importancia que pueda tener esta habilidad, pero un Liceo de Niñas no es el establecimiento que debía dársele preferencia sobre los ramos que desarrollan el carácter." ${ }^{\prime 35}$

La educación estaba planificada para acentuar los signos de la clase y el género, situando a cada quien en un orden y espacio, Teresa Prats de Sarraeta señala: "Su objetivo en primer término, la educación lógica de la mujer, tendente a sostener la armonía de los sexos i la solidez de los vínculos morales en la familia, i luego proporcionar a aquellas que lo necesitan o tienen especial vocación, una esfera de labor adecuada a su sexo o a su nivel social, a la vez que lucrativa y honrosa." 36

Fuera de los ramos de adornos la higiene y la salud corporal se establecieron como parámetros de formación. Los médicos enfatizaban la importancia de la 'jimnacia' en los planes educacionales como deber de carácter nacional. En el informe del doctor 
Murillo se señalan: "Al paso que hasta ahora hemos marchado, con el descuido que nos ha caracterizado, con la indolencia con que memos mirado tan altos como interesantes problemas, vamos al decaimiento progresivo de la juventud i mas de una vez permitido que ibamos a hacer un gran hospicio de una bella $\mathrm{i}$ viril nación." ${ }^{37}$

El movimiento médico adquirió cada vez más importancia en los círculos gobernantes, influencia que se acrecentaría durante el gobierno de Balmaceda y la inauguración del primer encuentro médico en 1889. Un informe del doctor Murillo solicitado por el Ministerio de Instrucción Pública daba cuenta de esta situación: “No Hace Mucho tiempo el señor Ministro de Instrucción Pública acudió a la Facultad de Medicina para proponerles e interesarla en el estudio de algunas cuestiones de palpitante interés. La facultad aceptó con placer tal invitacion i quedó empeñada en hacer lo que estuviera de su parte para llenar los deseos del alto funcionario..." 38

Sin embargo, los temas de salubridad e higiene permanecieron fuera de las preocupaciones centrales del Estado, de esto da cuenta un decreto de 1885 que situaba a los hospitales provinciales bajo la administración de los vecinos notables y las Juntas Locales de Beneficencia.

Únicamente al iniciar el siglo XX este movimiento obtuvo sus primeros resultados al fundar en 1901 el Patronato Nacional de la Infancia, destinado al cuidado y protección de menores indigentes y de sus madres y el 15 de noviembre de 1913, Manuel Arriarán, con la colaboración de Emilie Wait, levantaron el segundo hospital de niños de Valparaíso. El 14 de junio de 1920 se creó el departamento de sanidad, una propuesta curiosamente surgida desde el Ministerio de Guerra. En 1929 Ibañez estableció la Junta de Auxilio Escolar que disponía de un cinco por ciento de los ingresos de los municipios. Poco a poco la Iglesia y la aristocracia fueron perdiendo protagonismo y el Estado se hizo cargo del asunto. Entre las décadas de 1930 y 1940 el Estado inició una política de inclusión de "lo popular." Esto consistió en la implementación de una serie de mecanismos de reconocimiento, mediante el accionar de agentes públicas denominadas Visitadoras Sociales, cuyo principal objetivo era la rearticulación del cuerpo y la sexualidad 
popular dentro de los márgenes legales del sistema social. En este proceso de constitución del Estado-Nación las Visitadoras Sociales adquirieron un doble estatus, en cuanto a género se mantuvieron cercanas a lo femenino, lo agráfago, lo popular y la sexualidad; pero del mismo modo en cuanto Agentes se constituyeron en portadoras de un nuevo pacto escritural entre lo popular y el Estado.

\section{Conclusiones}

El Higienismo en Chile se estableció como parámetro y coordenada de imaginarios corporales y espaciales, constituyendo, a la vez, una casta especializada médica denominada por algunos autores como Intelligentzia Médica y que influyó, a partir de la década de 1870, notoriamente en las nuevas estructuras legales, educacionales y morales en nuestro país. En este Sistema, las mujeres quedaron excluidas del ejercicio de las labores "nobles" relacionadas con la producción, siendo relegadas a los ámbitos privados y la "reproducción," muchas fueron recluidas en casas de rehabilitación o sometidas en sus propios hogares a un corpus de aprendizajes referentes al cuerpo. El Sistema Liberal se asoció con los saberes médicos higienistas en un proyecto de ordenación del sistema corpóreo/espacial, instituyendo agencias correccionales que disciplinaran a los sujetos abyectos.

Al contrato social roussoniano que garantizaba los derechos inalienables de una parte de la masculinidad no se agregó un necesario "Contrato Sexual" que garantizara, del mismo modo, los derechos de la mayor parte de la ciudadanía republicana. El lenguaje y los discursos, tal como señala Violi, fueron ajenos a una identidad política femenina, negando a éstas la apropiación de sus propios cuerpos y siendo sometidas a un imaginario afectivo/sexual patriarcal. La "nueva república" excluyó a las mujeres del ejercicio político siendo forzadas a asumir una concepción del espacio y de sus propios cuerpos coercitiva y ajena, incluso muchas mujeres intelectuales reprodujeron este Sistema a falta de otras claves identitarias disponibles. El higienismo modeló el cuerpo femenino y lo situó 
en el vértice del sistema como reproductor físico y simbólico del discurso dominante, del mismo modo que constituyó un ideario político de Estado-Nación burgués, patriarcal y heterosexista.

\section{Notas}

1 Licenciado en historia, Magíster en Estudios de Género y Cultura de la Universidad de Chile, Becario CONICYT en programa de Doctorado en Estudios Americanos, Universidad de Santiago de Chile.

2 Revelación anticipada, ya que no fue hasta 1871 que Darwin expuso el principio evolutivo del hombre en su obra The Descent of Man and Selection in relation to Sex. Texto de apoyo para el estudio de las Ciencias Naturales del Instituto Nacional. 1866.

3 Revista Médica de Santiago, Volumen I, 1872, página 8.

4 La Revista Médica fue fundada por 33 estudiantes de la Facultad de Medicina de la Universidad de Chile junto a 13 médicos, la mayoría profesores de la Escuela de Medicina. Otro de los medios de difusión fue la Revista de Higiene de Santiago fundada en 1894, órgano representativo de el Consejo Superior de Higiene Pública.

5 Revista Médica de Santiago, Volumen I, 1872, página 8.

6 Otros médicos ilustres que formaron parte de la Sociedad Médica de Santiago fueron Rodolfo Amando Philippi, Lorenzo Sazié, José Joaquín Aguirre, Augusto Orrego Luco, Roberto del Río.

7 Revista Médica de Santiago, Volumen I, 1872 página 6.

8 Díaz, Eloísa, Breves observaciones sobre la aparición de la pubertad en la mujer Chilena $i$ de las predisposiciones patolójicas propias del sexo. Memorias para optar al grado de Licenciada en la Escuela de Medicina de la Universidad de Chile. 25 de diciembre de 1886.

9 Ibidem.

10 El trabajo, en este sentido, concibe una triple función: función productiva, función simbólica y función de domesticación o disciplinaria. La función productiva concierne a los cuerpos sanos y reproductivos en tanto los cuerpos incapacitados; locos, enfermos y anormales deben ser disciplinados mediante el trabajo y el castigo, ingresando de esta forma en el sistema productivo.

11 En este Sistema la arquitectura desempeñó un rol determinante, todos los grandes proyectos de vigilancia y de encierro retornaban al modelo de control y al "Panóptico"; un edificio circular dividido en celdas con dos ventanas; una abierta hacia el interior que se corresponde con las ventanas de una torre de vigilancia. 
12 Institución que había sido fundada en 1726 como asilo para meretrices.

13 Gaceta de los Tribunales, No 109, de marzo, de 1844. Cita de Cárdenas, Mario, Grupos marginados en los inicios de la era republicana... P. 50 .

14 Allende, Ramón, Discurso de incorporación a la Facultad de Ciencias Médicas de la Universidad de Chile, 1 de noviembre de 1885, Anales de la Universidad de Chile, tomo XXVII segundo semestre de 1865, pág. 528. Consultado en "Memoria Chilena," diciembre del año 2008, http://www.memoriachilena.cl/archivos2/pdfs / MC0023712.pdf

15 Ver Cárdenas, Mario, Grupos marginados en los inicios de la era republicana: Vagabundo mendigos e indigentes, Cuadernos de Historia $n^{\circ}$ 11, Departamento de Ciencias históricas, Facultad de Filosofía y Humanidades, Universidad de Chile, Santiago de Chile, diciembre de 1991.

1619 de octubre, 1842. Sesiones de los Cuerpos Legislativos. Tomo XXX, P. 433. Cárdenas Mario, Grupos marginales en... op. cit. P. 59.

17 Allende, Ramón, Discurso de incorporación a la Facultad de Ciencias Médicas de la Universidad de Chile, op. cit., pág. 526.

18 Allende, Ramón, Discurso de incorporación a la Facultad de Ciencias Médicas de la Universidad de Chile, op., cit., pág. 527.

19 Ibidem.

20 Ibidem.

21 El Despertar... nº 1985 domingo 24 de abril de 1921.

22 El Despertar de los Trabajadores. n 2021 domingo 25 de junio de 1921.

23 El Despertar de los Trabajadores de Iquique. No 2739 miércoles 30 de abril de 1924.

24 Allende, Ramón, Discurso de incorporación a la Facultad de Ciencias Médicas de la Universidad de Chile, op. cit., pág.527.

25 Calvo Makenna, Luis, Lo que deben saber las madres para criar a sus niños. Primer Congreso de Protección de la Infancia, Tomo único, Imprenta Barcelona, Santiago de Chile, 1913, pág. 1. En Memoria Chilena consultada en diciembre del año 2008, pág.1. http: / / www. memoriachilena.cl/archivos2/pdfs/MC0023729.pdf

26 Körner, Victor, Protección de las Madres durante el embarazo, el parto, el pueperio, y su influencia sobre la mortalidad infantil. Tomo único, Imprenta Barcelona, Santiago de Chile, 1913, pág. 1. En Memoria Chilena consultada en diciembre del año 2008, pág. 1. http:/ / www.memoriachilena.cl/archivos2/pdfs/MC0023729.pdf

27 Simon, Robert, Reducción de la mortalidad infantil del 1er año por la asistencia á las madres antes, durante y después del parto. Primer Congreso de Protección de la Infancia, Tomo único, Imprenta Barcelona, Santiago de Chile, 1913, pág. 1. En Memoria Chilena consulta- 
da en diciembre del año 2008, pág.1. http: / / www.memoriachilena. cl/archivos2/pdfs/MC0023729.pdf

28 Vicuña Mackenna, Benjamín; La Policía de Seguridad en las grandes ciudades modernas. op. cit. Pp. 11-12.

29 Amunátegui, Miguel Luis, Estudios sobre Instrucción Pública, Tomo II, Santiago, Imprenta Nacional, 1894, p. 261.

30 Díaz, Eloísa, Breves observaciones sobre la aparición de la pubertad en la mujer Chilena $i$ de las predisposiciones patolójicas propias del sexo. Memorias para optar al grado de Licenciada en la Escuela de Medicina de la Universidad de Chile. 25 de diciembre de 1886.

31 Informe del Doctor Murillo, Revista Médica, Volumen I, 1872 pág. 30.

32 En 1856 Manuel Montt constituye entre grupos de notables aristócratas la Sociedad de Instrucción Primaria con el fin de desarrollar el ámbito educacional público, pero es sólo a partir de la Ley Orgánica de la Enseñanza de 1860, que el Estado otorga los primeros indicios en este ámbito.

33 Díaz, Eloísa, Breves observaciones sobre la aparición de la pubertad en la mujer Chilena $i$ de las predisposiciones patolójicas propias del sexo. Memorias para optar al grado de Licenciada en la Escuela de Medicina de la Universidad de Chile. 25 de diciembre de 1886.

34 Prospecto del Liceo de Niñas $N^{o} 2$ de Santiago, Redactado en conformidad a los acuerdos de la Junta de Vigilancia. Imprenta, Litografía i Encuadernación Barcelona, Santiago, 1900, Pp. 14-15.

35 Gremler, Juana, Memoria del Liceo 1, A. M. E. Vol.1588.

36 Prats de Sarraeta, Teresa, Proyecto de Reorganización de los Liceos de Niñas de la República, Imprenta i Encuadernación Universitaria, Santiago, 1905, p.30.

37 Informe del Doctor Murillo, Revista Médica, Volumen I, 1872 pág. 31.

38 Informe del Doctor Murillo, Revista Médica, Volumen I, 1872, pág. 29. 


\section{Bibliografía}

Allende, Ramón. Discurso de incorporación a la Facultad de Ciencias Médicas de la Universidad de Chile. 1 de noviembre de 1885, Anales de la Universidad de Chile, tomo XXVII segundo semestre de 1865, pág. 528. Consultado en "Memoria Chilena." http:// www.memoriachilena.cl/archivos2/pdfs/MC0023712.pdf

Amunátegui, Miguel Luis. Estudios sobre Instrucción Pública, Tomo II, Santiago: Imprenta Nacional.

Arrom, Silvia Marina. Las mujeres de la ciudad de México, 1790-1857, Madrid: Siglo XXI ediciones. (1985).

Calvo Makenna, Luis. Lo que deben saber las madres para criar a sus niños. Primer Congreso de Protección de la Infancia, Tomo único, Santiago de Chile: Imprenta Barcelona, 1913, pág. 1. En Memoria Chilena consultada en diciembre del año 2008, pág.1. http://www.memoriachilena.cl/archivos2/pdfs/MC0023729.pdf

Cárdenas, Mario. "Grupos marginados en los inicios de la era republicana: Vagabundo mendigos e indigentes." Cuadernos de Historia $\mathrm{N}^{\circ}$ 11, diciembre, Santiago: Universidad de Chile, 1991.

Cavieres, Paola y Eliana Pérez, Carola Sepúlveda, profesora guía Sofía Correa. "Cocinar, Tejer, Coser y Bordar: La Acción de los Primeros Liceo Femeninos Fiscales de Santiago en el Reforzamiento del Rol Tradicional de la Mujer (1894-1912)." Tesis para optar al grado de Licenciatura en Historia, Universidad de Santiago de Chile, 2004.

Del Pilar Sinués, María. "Estudios acerca de la Educación de la Mujer," Madrid, 1876.

Díaz, Eloísa. Breves observaciones sobre la aparicion de la pubertad en la mujer Chilena i de las predisposiciones patolojicas propias del sexo. Memorias para optar al grado de Licenciada en la Escuela de Medicina de la Universidad de Chile. 25 de diciembre de 1886.

Foucault, Michel. Las tecnologías del yo. Barcelona: Editorial Paidós Ibérica. S.A., 1995.

----- Historia de la sexualidad. El uso de los placeres. México: Siglo XXI, 1991.

----- Historia de la sexualidad, La inquietud de Sí. México: Siglo XXI, 1991.

“La Gouvernementalité." En Dits et Écrits, Tome IV, pp. 641-6 42. Citado en Lazzarato, Mauricio. "Del Biopoder a la Biopolítica." En Revista Multitudes No 1, París, marzo, 2000. Disponible en: http:/ / www.sindominio.net/arkitzean/otrascosas/lazzarato

"El ojo del Poder" en Entrevista con Michel Foucault. En Jeremías Bentham. 'El Panóptico'. Barcelona: Ed. La Piqueta, 1980. Traducción de Julia Varela y Fernando Alvarez-Uría. Disponible en: http// rie.cl/?a=1009

Gentilini, Bernardo. “El Libro de la Mujer. Como Cristiana, Esposa, Madre, 
Educadora y Apóstol," Santiago: Apostolado de la Prensa, 1928. Gremler, Juana, Memoria del Liceo 1, A. M. E. Vol.1588.

Körner, Victor, Protección de las Madres durante el embarazo, el parto, el puerperio, y su influencia sobre la mortalidad infantil. Tomo único, Santiago de Chile: Imprenta Barcelona, 1913, pág. 1. En Memoria Chilena. http:/ / www.memoriachilena.cl/archivos2/pdfs/MC0023729.pdf

Labarca, Amanda. "Nuestras actividades femeninas" (1923). En ¿A dónde va la mujer, Santiago: Ediciones Extra, 1934.

Lavrin, Asunción. Sexualidad y matrimonio en la América Hispana. México: Ediciones Grijalbo, 1989.

Márquez Bretón, Bernardo. Orígenes del Darwinismo en Chile. Santiago de Chile: Editorial Andrés Bello, 1982.

Montecino, Sonia. "Identidades de género en América Latina: Mestizajes, sacrificios, simultaneidades." En Género e identidad. Ensayos sobre lo femenino y lo masculino, Bogotá: Tercer Mundo editores, 1995.

Murillo. Informe Revista Médica, Volumen I, 1872.

Prats de Sarraeta, Teresa. Proyecto de Reorganización de los Liceos de Niñas de la República, Santiago: Imprenta i Encuadernación Universitaria, 1905.

Prospecto del Liceo de Niñas $N^{o} 2$ de Santiago, Redactado en conformidad a los acuerdos de la Junta de Vigilancia. Santiago: Imprenta, Litografía i Encuadernación Barcelona, 1900.

Revista Médica de Santiago, Volumen I, 1872.

Richard, Nelly y Patricia Violi. “¿Tiene Sexo la Escritura?.” En Masculino/Femenino. Santiago: Ed Francisco Zegers, 1993.

Simon, Robert. Reducción de la mortalidad infantil del 1er año por la asistencia á las madres antes, durante y después del parto. Primer Congreso de Protección de la Infancia, Tomo único, Santiago de Chile: Imprenta Barcelona, 1913, pág. 1. En Memoria Chilena. http://www.memoriachilena.cl/archivos2/pdfs/MC0023729.pdf

Stuven, Ana María. “Republicanismo y liberalismo en la primera mitad del siglo XIX: ¿hubo proyecto liberal en Chile?." Comps. Manuel Loyola y Sergio Grez, Los proyectos nacionales y el pensamiento politico y social chileno del siglo XIX. Santiago: Ediciones UCSH, 2002.

Vicuña, Manuel. La Belle Epoque Chilena. Santiago: Editorial Sudamericana, 2001.

Vicuña Mackenna, Benjamín. Memoria sobre el Sistema Penitenciario en General y su mejor aplicación en Chile. Santiago: Impreso Dirección General de Prisiones, Universidad de Chile, 1941.

----- La Policía de Seguridad en las grandes ciudades modernas, Santiago: Imprenta La República, 1875.

----- “Discursos Parlamentarios I." En Obras Completas. Santiago: Dirección General de Prisiones, Volumen XII, pp. 600-612, 1939.

“Discursos Parlamentarios III." En Obras Completas, Santiago: Dirección General de Prisiones, Volumen XIV, pp. 330-333 y 336 -341, 1939. 
----- Los Lisperger y La Quintrala. Santiago: Editorial Sudamericana, 2001.

Violi, Patricia. El Infinito Singular, Madrid: Ediciones Cátedra, 1991.

VV.AA., El Despertar de los Trabajadores de Iquique, órgano oficial del Partido Obrero Socialista, Iquique, 1921-1925.

Zegarra, Margarita. Del Silencio a la Palabra Mujeres Peruanas S. XIX-XX, Ediciones Flora Tristán, 1992.

------ Mujeres y Género en la Historia del Perú. Ed. Margarita Zegarra. Lima, CENDOC-Mujer. 